\title{
Werner Gumpel \\ Migration und Immigration als politisches, wirtschaftliches und soziales Problem in Russland
}

\section{Einleitung}

Russland hat ein demographisches Problem: Die Bevölkerung des Landes schrumpft. Schätzungen zufolge wird sie von derzeit 143 Millionen bis zum Jahr 2050 auf 100 Millionen sinken. ${ }^{1}$ Einige Regionen entvölkern sich, da die Menschen wegen Arbeitsplatzmangels und einer mangelhaften Infrastruktur, aber auch wegen der herrschenden klimatischen Bedingungen, in andere Gebiete Russlands oder ins Ausland abwandern. Dabei erweist sich die Auswanderung insofern als besonders problematisch, als es vor allem gut ausgebildete Arbeitskräfte sind, die ein Leben in einem anderen Land vorziehen. Andererseits erfolgt eine im Grunde erwünschte Zuwanderung aus dem Ausland, die aber den Abfluss von qualifizierten Arbeitskräften und den natürlichen Bevölkerungsrückgang durch Geburtenentwicklung und Todesfälle nicht kompensieren kann. Die Immigranten sind in ihrer überwiegenden Mehrzahl Menschen mit geringer oder keiner Qualifikation. Der größte Teil von ihnen lebt illegal im Land. Es ist daher zu unterscheiden zwischen innerer Migration und regulärer und irregulärer (illegaler) Immigration. Alle drei Formen schaffen für das Land eine Vielzahl von Problemen sowohl politischer als auch wirtschaftlicher und sozialer Dimension. Sie bedeuten nicht zuletzt auch eine sicherheitspolitische Herausforderung für das Land. In den nachfolgenden Ausführungen wird zunächst die Bedeutung der russischen Binnenmigration mit ihren Ursachen und Wirkungen untersucht, um im nachfolgenden Teil das Ausmaß und die Folgen insbesondere der illegalen Migration zu analysieren.

\section{Innere Migration}

Die innere Migration in Russland wird von mehreren Faktoren determiniert. Über allem steht die demographische Entwicklung im Land. Die Anzahl der Bewohner des Landes zeigt, wie oben dargelegt, seit Jahren eine abnehmende Tendenz. Ursächlich ist eine rückläufige Geburtenhäufigkeit. Die Sterbeziffern übertreffen die Geburtenzahlen erheblich. Gleichzeitig nimmt, wie erwähnt, auf Grund der herrschenden Lebens- und Arbeitsbedingungen der Drang zur Auswanderung zu. Derzeit erlebt Russland die stärkste Auswanderung seit 1917. In den letzten zehn Jahren haben laut»Inopressa« mehr

1 Izvestija, 28. 1. 2005. Die Izvestija vom 22. 3. 2006 sieht die Entwicklung etwas optimistischer. Danach sinkt die Bevölkerungszahl bis zum Jahr 2050 »nur« auf 113 Millionen. 
als 1,25 Millionen Menschen das Land verlassen. Die jährliche Emigration wird auf 100 - 150 tausend Personen geschätzt, mit steigender Tendenz. Neben der Unzufriedenheit mit der politischen Lage ist für die qualifizierten Arbeitskräfte der Ärger über die veraltete, oft seit Jahrzehnten nicht erneuerte Kapitalausstattung der Unternehmen, sowie die überbordende Korruption auf allen Ebenen der Administration und der Wirtschaft und, für die Unternehmer, die Unmöglichkeit ein Unternehmen auf legale Weise zu führen, der Anstoß, einen Neubeginn im Ausland zu suchen. ${ }^{2}$ Laut einer Umfrage des »Analytischen Zentrums Levada« erwägen etwa 30 Prozent der Befragten, Russland zu verlassen. Drei bis vier Millionen beginnen bereits mit entsprechenden Vorbereitungen, indem sie die erforderlichen Dokumente sammeln, und Verbindungen ins Ausland herstellen. Sie sehen, so die Soziologen des Zentrums, für die nächsten zwölf Jahre (voraussichtliche Dauer der erwarteten Präsidentschaft von Putin) auf dem Territorium Russlands keine Perspektiven für sich. ${ }^{3}$ Beliebteste Zielländer der Auswanderer sind Deutschland, die USA und Großbritannien. ${ }^{4}$

$\mathrm{Da}$ die Geburtenziffern bzw. die Sterblichkeit sich in den verschiedenen Regionen unterscheiden, und die Abwanderung der gebildeten Personen vorwiegend die industriell entwickelten Gebiete betrifft, sind die Auswirkungen regional sehr unterschiedlich. Die nach außen gerichtete Migration wird in ihren Wirkungen durch die Migration aus den wenig erschlossenen und den klimatisch benachteiligten Regionen des Landes in die entwickelten Gebiete insbesondere des europäischen Landesteils, verstärkt. Ganzen Landstrichen droht eine zunehmende Entvölkerung. Das betrifft, wie noch zu zeigen ist, insbesondere den östlichen Landesteil, also Ostsibirien und den sogenannten Fernen Osten.

Die demographische Entwicklung und die faktische Entleerung ganzer Gebiete stellt Russland vor eine Vielzahl von schwerwiegenden wirtschaftlichen und sozialen Problemen. Sie zu lösen erfordert einen erheblichen Kapitaleinsatz. An erster Stelle nämlich müssen in Russland Bedingungen geschaffen werden, die den Wunsch, die heimatliche Region oder gar das Land zu verlassen, gar nicht erst aufkommen lassen. Für die entleerten Gebiete müssen Anreize zur Zu- bzw. Rückwanderung geschaffen werden. Hierzu gehören die Errichtung einer effizienten verkehrswirtschaftlichen und sozialen Infrastruktur, und die Verbesserung der Umweltbedingungen. Es müssen neue Bahnlinien und Straßen errichtet und ein verfügbares Gesundheitswesen, sowie Schulen und andere Bildungseinrichtungen geschaffen werden. Damit verbunden muss die Schaffung von Arbeitsplätzen sein. Das ergibt sich auch aus der Notwendigkeit, die im Osten des Landes gelegenen Erdöl- und Erdgasressourcen zu erschließen, da die im westlichen Landesteil und im Ural gelegenen Vorkommen langsam der Erschöpfung entgegen gehen. In der Zeitung »Izvestija« geht man davon aus, dass 17 bis 18 Millionen Menschen nach dem Fernen Osten des Landes transferiert werden müssten um den wirtschaftlichen Erfordernissen gerecht werden zu können. ${ }^{5}$ Doch wie soll dieses Ziel ohne Zwangsmaßnah-

2 www.newsru.com/russia/16nov 2011/svali.

3 www.levada.ru/print/17-11-2011/ottok chelovecheskogo-kapitala (Abfluss von Humankapital.).

4 www.expert.ru/2011/11/16/razgovor-pro.

5 Izvestia, 22.3.2006.

ZfP 59. Jg. 3/2012 
men, wie sie in der Zeit Stalins oder bei der sogenannten Neulanderschließung in den fünfziger Jahren, angewandt wurden, realisiert werden?

\section{Immigration - Lösung oder Verschärfung der bestehenden Probleme?}

Die bisherigen Ausführungen zeigen, dass die russische Regierung an einer "Auffüllung « der russischen Bevölkerung durch Immigration interessiert sein muss. Dass dies auf vielerlei erwünschte und unerwünschte Weise erfolgen kann, ist das, was für die Regierenden ein Problem höchster Komplexität darstellt.

\section{a. Legale Immigration}

Zu den erwünschten Migranten gehören zweifellos jene Russen, die ihren schon zu Sowjetzeiten begründeten Wohnsitz in den ehemaligen Sowjetrepubliken verlassen, weil sie dort diskriminiert oder anderweitig benachteiligt werden, bzw. unter den neuen postsowjetischen Bedingungen ihren Arbeitsplatz verloren haben. Sie sprechen die Landessprache und sind, obwohl in einer nichtrussischen Sowjetrepublik aufgewachsen, in der eigenen Ethnie sozialisiert und zumeist gut ausgebildet. Es ist lediglich eine Frage der Zeit, dass sie am neuen Wohnort integriert werden. Schwierigkeiten bringt hier allerdings die Versorgung mit Wohnraum und Arbeitsplätzen. In Regionen mit Wohnraummangel und Arbeitslosigkeit führt dies zu mangelnder Akzeptanz bei der ansässigen Bevölkerung und entsprechenden Friktionen. Es gilt allerdings zu berücksichtigen, dass Arbeitslosigkeit und Armut auf dem Land und in den Randgebieten vieler Städte noch immer groß sind. In den großen Städten gibt es zunehmend ein »Elendsproletariat«, das nicht zuletzt eine Folge der Zuwanderung aus den ehemaligen Sowjetrepubliken ist.

Legal ist auch die Zuwanderung aus verschiedenen ehemaligen Sowjetrepubliken, soweit sie mit offizieller Genehmigung erfolgt, oder offizielle Aufenthaltsgenehmigungen für die Orte der Niederlassung erteilt werden. Ein wirkliches Konzept zur Lenkung der Migration besteht noch nicht. Doch hat die Eurasische Wirtschaftskommission (EWK), die durch einen am 18. November 2011 von den Präsidenten der Mitgliedsländer unterzeichneten Vertrag an die Stelle der Zollunion von Russland, Weißrussland und der Ukraine getreten ist und zu einer Vertiefung der Integration dieser Staaten und zur Schaffung gemeinsamer Strukturen führen soll, beschlossen, im Rahmen des von ihr angestrebten Eurasischen Wirtschaftsraums auch den Bereich der Arbeitsmigration unter den Mitgliedsstaaten zu regeln. Da das Ziel der EWK die Schaffung eines Einheitlichen Eurasischen Wirtschaftsraumes ist, bedeutet der am 12. Januar 2012 in Kraft getretene Vertrag in seiner Konsequenz auch die Freizügigkeit für die Arbeitskräfte innerhalb des Integrationsgebietes. ${ }^{6}$ Damit wäre allerdings erst ein geringer Teil des Problems der Arbeitskräftemigration in geregelte Bahnen geleitet. Die in diesem Hinblick wichtigeren

6 Novikova, Anastasija: Evraskie komissari polutschat status federnal'nych ministrov (Die eurasischen Kommissare erhalten den Status von Bundesministern), in. Izvestija, 17. 11. 2011 (www.izvestija.ru/news/507125.). 
zentralasiatischen Republiken sind damit nicht erfasst. Allerdings erhofft die russische Seite, dass auch sie eines Tages dem Einheitlichen Eurasischen Wirtschaftsraum beitreten.

Russland selbst versucht für die irregulär eingewanderten Arbeitskräfte Anreize für die Erlangung von Aufenthaltsgenehmigungen und damit zur Legalisierung ihrer Arbeitsverhältnisse zu schaffen. So wird die Mitgliedschaft in der Rentenversicherung (»Pensionnyi fond «) und der Empfang von Renten ermöglicht, wenn die Betroffenen mindestens sechs Monate im Land arbeiten. Diese Regelung ist seit dem 1. Januar 2012 wirksam. Da die Arbeitgeber jedoch für nur kurzzeitig Beschäftigte weder Abführungen in die Rentenversicherung noch in die Sozial- und Krankenversicherung leisten müssen, ziehen sie es vor, irreguläre Migranten zu beschäftigen. ${ }^{7}$ In der Realität wird die neue Regelung nur sehr begrenzte Auswirkungen zeitigen. Die irreguläre bzw. illegale Einwanderung wird daher auch weiterhin erhalten bleiben. Allerdings wird sie sich nicht als eine Einwanderung in das Sozialsystem erweisen, da Illegale keinerlei Sozialleistungen empfangen können.

\section{b. Illegale Immigration}

Obwohl Russland eine zwar abnehmende, vor allem regional aber hohe Arbeitslosigkeit aufweist, ist der Anreiz zur Immigration hoch (Arbeitslosigkeit im November 2011: 7,6 Prozent, auf dem Land: 9,5 Prozent). ${ }^{8}$ Der Mangel an Arbeitsplätzen ist in den Ländern, aus denen die Zuwanderung erfolgt, beträchtlich größer. Auch der Lebensstandard ist in der Russländischen Föderation (RF) höher. Die Mehrzahl der »Gastarbeitery« kommt aus Zentralasien, an erster Stelle aus Usbekistan, gefolgt von Aserbaidschan, Kirgistan, Turkmenistan. In der Anzahl der Zuwanderer steht an zweiter Stelle allerdings die Ukraine, gefolgt von Armenien. ${ }^{9}$ Laut russischen Quellen gibt es derzeit (Zahl für November 2010) circa sieben Millionen Arbeitsmigranten in Russland. Davon arbeiten drei Millionen illegal. Von den vier Millionen »Legalen« zahlen nur die wenigsten Steuern, da sie es verstehen, die bestehenden Schlupflöcher in der russischen Gesetzgebung zu nutzen. So arbeiten die Ukrainer und die Mehrzahl der Moldawier jeweils nur drei Monate im Land, um nach kurzer Zeit erneut für drei Monate einzureisen. ${ }^{10}$ Es besteht daher eine Fluktuation. Die Mehrzahl der Migranten wird in der Schattenwirtschaft tätig. Verlässliche Statistiken über die Zahl der »Gastarbeitery « gibt es aus verständlichen Gründen allerdings nicht. So schätzt die Weltbank deren Zahl für das Jahr 2010 auf 12,3 Millionen. Sollte diese Zahl richtig sein, stünde Russland in Hinblick auf die Zahl der Migranten an zweiter Stelle in der Welt. ${ }^{11}$ Auch diese Zahl scheint aber zu niedrig gegriffen.

7 Pensii dlja migrantov stanut ralnostju (Pensionen für Migranten werden Realität). www.bigness.ru/ ?area=articleItim\&id=13040 (25. 11. 2011.).

8 Slushba Gosudarstvennoj Statistiki (Staatliche Amt für Statistik) Januar 2012.

9 www.izvestija.ru/news/news229977 (28. 01.2010.).

10 www.newsru.com/russia/23.nov 2010/migranty_print.html.

11 Ebenda.

ZfP 59. Jg. 3/2012 


\section{c. Ausmaß und Formen der irregulären Migration und deren Wirkungen}

Drei Formen der irregulären Immigration können unterschieden werden. Es sind dies

- die Immigration aus dem sogen. »nahen Ausland «, das sind die unabhängig gewordenen ehemaligen Sowjetrepubliken. Hier handelt es sich in erster Linie um die Zuwanderer aus Zentralasien, der Ukraine, dem Südkaukasus und dem Baltikum;

- die Immigration aus den Problemrepubliken der russländischen Föderation im Kaukasus (Tschetschenien, Inguschien, Dagestan)

- die Immigration aus dem »Fernen Ausland « (vornehmlich China und Vietnam sowie in minderer Zahl aus einigen Ländern Afrikas).

Aus ökonomischer Sicht ist die Zuwanderung, wie an anderer Stelle erwähnt, durchaus sinnvoll und erwünscht um den Schrumpfungsprozess der russischen Bevölkerung zu kompensieren. Ohne die »Gastarbeitery « würden auf dem Arbeitsmarkt erhebliche Lücken und damit Friktionen im Wirtschaftsablauf auftreten, die sich auf das Wirtschaftswachstum des Landes auswirken würden. Sie arbeiten vorwiegend in Bereichen, in denen sie als unqualifizierte Arbeitskräfte eingesetzt werden können. Sie sind aber auch in starkem Maße als Händler tätig und beherrschen die lokalen Märkte. Da sie, wie gezeigt, zu einem wesentlichen Teil ohne Arbeitsgenehmigung tätig werden, sind sie für die russischen Unternehmer und Arbeitgeber ein leicht zu manipulierendes Ausbeutungsobjekt. Sie arbeiten großenteils zu Löhnen, die weit unter denen liegen, die für reguläre Arbeitnehmer gezahlt werden. Oftmals werden sie um ihren Lohn betrogen. Auf Grund der Illegalität ihres Aufenthaltsstatus haben sie keine Möglichkeit sich zu wehren und Außenstände einzutreiben. Andererseits tragen sie zur Mehrung des Sozialprodukts und der internationalen Wettbewerbsfähigkeit des Landes bei. Gastarbeiter sind mobiler und flexibler als die einheimische Bevölkerung und können sich dementsprechend den jeweiligen Erfordernissen des Arbeitsmarktes schnell anpassen. Für die Entsenderländer sind dagegen ihre Remissen von erheblicher wirtschaftlicher Bedeutung. Der International Fund of Agricultural Development hat berechnet, dass in den Jahren 2006 und 2007 die Überweisungen etwa 31,4 bzw. 36,7 Prozent des Bruttoinlandsprodukts der betroffenen Länder entsprachen, in Usbekistan waren es 17 Prozent im Jahr 2006. ${ }^{12}$ Der Präsident der Nationalbank Tadshikistans verlautbarte, dass Migranten im Jahr 2011 fast 2,96 Milliarden Dollar aus Russland in sein Land transferiert haben. Das entspricht 45,4 Prozent des dortigen Bruttoinlandsprodukts. ${ }^{13}$ Für die Wirtschaft der Heimatländer der Auslandsarbeiter stellen daher deren Remissen eine fühlbare Entlastung dar.

Zwar wurde im Jahre 2006 für zehn ehemalige Teilrepubliken der UdSSR der Visumzwang für die Einreise in die RF aufgehoben und die Erteilung einer Aufenthalts- und Arbeitserlaubnis erleichtert, nach wie vor sind die bürokratischen Hürden jedoch hoch. Um die Arbeitskosten niedrig halten zu können ist die Privatwirtschaft jedoch an einer

12 Saidazimova, Gulnoza: Central Asia: Labor Migrants Face Abuse, Xenophobia. Radio Free Europe, 22. 10. 2007 (www.rferl.net/featuresarticle//2007/10/75118827-D89-7D89-462E.).

13 Migranty is Tadshikistana pereveli na rodinu is Rossii potschti 3 Mrd. Dollar (Migranten aus Tadshikistan tranferierten aus Russland fast 3 Mrd. Dollar); Newsru.com.finance/19jan2012/ tadjikistan. 
solchen Liberalisierung nur wenig interessiert. Dies gilt umso mehr, als illegal tätige Arbeitskräfte in Zeiten einer Rezession problemlos entlassen werden können, was bei legal tätigen Arbeitskräften nicht ohne weiteres möglich ist. Für den russischen Staat bedeuten die Illegalen allerdings insofern eine Belastung, als sie, wie gezeigt, die Entrichtung von Steuern, wo dies möglich ist, umgehen, also keine Entlastung für den Staatshaushalt bringen, andererseits aber die vorhandene Infrastruktur, insbesondere die Verkehrsinfrastruktur, aber auch (wo dies auf Grund ihres Status möglich ist) die soziale Infrastruktur und die Umweltressourcen nutzen. Belastend wirkt sich auch in vielerlei Hinsicht ihre Nachfrage nach Wohnraum bzw. Unterbringungsmöglichkeiten aus. Besonders belastend ist jedoch die Verminderung der inneren Sicherheit des Landes.

\section{Migranten und innere Sicherbeit}

In Hinblick auf die innere Sicherheit sind vor allem zwei Problembereiche relevant. Das sind die Gefahren die auf Grund der hohen Durchsetzung des Landes mit Ausländern von ethnischen Auseinandersetzungen ausgehen, und zweitens die Probleme, die eine zunehmende Kriminalität bringt. Sind die ersteren einem stark ausgeprägten Nationalismus zuzuschreiben, so die letzteren der Tatsache, dass die Immigration in erster Linie eine Armutsmigration ist. Beide Bereiche sind jedoch eng mit einander verwoben.

Spätestens seit Eroberung des Kaukasus denken und handeln die Russen wie eine Kolonialmacht und pflegen gegenüber den dortigen Völkerschaften ein Gefühl der Überlegenheit. Gleiches gilt für die Völker Zentralasiens. Der russische Ethnozentrismus zeigt sich auch unverhüllt gegenüber den in der heutigen RF lebenden Menschen aus den betreffenden Regionen. Auch der von Stalin verfolgte "proletarische Internationalismus « hat dies nicht geändert. »In der Nationalitätenpolitik dominierte anstatt der harmonischen Zusammenführung der Interessen verschiedener Nationen und Völkerschaften ein großrussischer Chauvinismus, der mit dem staatlich gebilligten Antisemitismus und der Diskriminierung einer Reihe von Völkern einherging «, schreibt die Soziologin Tatjana Saslawskaja. ${ }^{14}$ Dieser fand unter Stalin seinen Ausdruck in der gezielten Dezimierung ganzer Völkerschaften. Dem »Holodomor « in der Unkraine, einer bewusst herbeigeführte Hungersnot, fielen sieben Millionen Ukrainer zum Opfer. Die Umsiedlung bzw. der Siedlungszwang für die Kasachen kostete ca. 40 Prozent des kasachischen Volkes das Leben und die Zwangsumsiedlung der Tschetschenen im Februar 1944 brachte ca. 50 Prozent der Bevölkerung den Tod. Die Behauptung Stalins, dass die Verfolgung der Tschetschenen und Inguschen wegen deren Kollaboration mit den Deutschen erfolge, war erlogen: Das Territorium der beiden Völker war nie von Deutschen besetzt. Ursächlich war vielmehr die Forderung der Tschetschenen und anderer kaukasischer Völker nach nationaler Unabhängigkeit. ${ }^{15}$

Auch heute zeigen die Russen eine tiefe Abneigung insbesondere gegenüber Zentralasiaten, Kaukasiern, und anderen Nationalitäten sowie Juden, die u. a. in diskriminie-

14 Saslavkaja, Tatjana: Die Gorbatschow-Strategie, Wien 1989, S 46.

15 www.kavkazcenter.net/eng/content/2011/02/23/13628_print.html (23..2 2011.).

ZfP 59. Jg. 3/2012 
renden Bezeichnungen ihren Ausdruck finden. Kaukasier und Zentralasiaten sind die »Schwarzen« oder die »Schwarzärsche«, Juden werden als »Shid « bezeichnet. Abwertende Bezeichnungen finden sich generell für Menschen »nichtslawischen Aussehens«, die auch gerne als »nichtrussische Teufel« tituliert werden, um hier nur einige der gängigen Bezeichnungen zu nennen. Die Perzeption von Überfremdung führt zu ethnischem Extremismus und Rassenhass. Sie »destabilisiert die Gesellschaft, bewirkt eine Schwächung des Staates, verstärkt bestehende Desintegrationsprozesse, propagiert separatistische Tendenzen und behindert damit die demokratische Entwicklung der Gesellschaft. Der Extremismus stellt eine Gefahr für die territoriale Unversehrtheit Russlands dar.«16 Nicht zu vergessen: In der Russländischen Föderation leben 182 verschiedene Völkerschaften.

Nach Schätzung von Menschenrechtsorganisationen wurden allein im ersten Halbjahr 2010 mehr als 90 Überfälle, begangen aus aggressiver Xenophobie, festgestellt. 22 Menschen wurden dabei getötet, 105 zum Teil schwer verletzt, der größte Teil davon in Moskau und im Bezirk Moskau, in St. Peterburg, im Gebiet von Altaj, in Vladivostok und in Jaroslavl. Doch die Dunkelziffer ist auch hier groß. Als »Naschi« (Unsrige) werden laut Umfragen Menschen nur dann betrachtet, wenn sie, unabhängig davon, wie lange sie an einem Ort leben, aus den zentralen Bezirken Russlands, Sibirien oder dem Fernen Osten zugezogen sind. Niemals aber könne man Migranten aus Aserbaidschan, Armenien oder Georgien zu den »Unsrigen« zählen. 68 Prozent der Befragten vertraten die Meinung, dass Immigranten aus Zentralasien niemals in Russland heimisch werden dürfen. Bewohner des Kaukasus würden für ewig Fremde bleiben. ${ }^{17}$ Immer häufiger und lauter ertönt der Ruf: »Russland den Russen«.

Die feindselige Einstellung der Russen gegenüber den anderen Völkerschaften des Staates zeigt die Brisanz des Nationalitätenproblems für die innere Sicherheit des Landes. Die Arbeitsmigration verstärkt diese Aversion. Wenn allein die Zahl der illegal in Moskau lebenden Angehörigen der ungeliebten Völkerschaften auf zwei Millionen geschätzt wird, lässt es sich leicht ermessen, dass die Abneigung ihnen gegenüber eher zu- als abnimmt. ${ }^{18}$

Je zahlreicher die Immigranten werden, desto stärker steigt ihr Anteil an der Kriminalität und mit ihr die den Zuwanderern entgegengebrachte Aggressivität. Meist sind es einzelne Personen oder kleine Gruppen von Menschen, die Opfer von Angriffen insbesondere von Skinheads oder anderen ausländerfeindlichen Jugendlichen werden. Dabei gilt der Hass nicht nur den Gastarbeitern aus Zentralasien und dem Kaukasus, sondern auch den im Land lebenden Menschen aus Afrika, China und anderen Staaten. Oft ist die Polizei zur Stelle, greift aber nicht ein. Ein Vorfall der wegen seines Ausmaßes dann doch zu einem Polizeieinsatz zur Wiederherstellung der Ordnung führte, waren die

16 Pogromy v Moskve: radikalism ili nastroj bol'schinstva (Die Pogrome in Moskau: Radikalismus oder Empfindung der Mehrheit). In: Nezavisimaja gazeta, 17. 12. 2010 (www.ng.ru/ moscow /2010-12-17/5we.html.).

17 Ebenda.

18 www.newsru.com/russia(23nov 2010/migranty_print.hrml. 
bürgerkriegsähnlichen Auseinandersetzungen am 11. Dezember des Jahres 2010 auf dem Manegeplatz in Moskau, die in der russischen Presse als Ausdruck des herrschenden »ethnischen Extremismus und Rassenhasses « bezeichnet wurden. ${ }^{19}$ Morde und Überfälle auf dem Boden von nationaler Feindseligkeit werden insbesondere in Moskau und St. Peterburg immer mehr zu einem Hauptproblem für die dortige Polizei. Ihre Zahl nahm in Moskau im Jahr 2008 im Vergleich zu 2007 um 300 Prozent zu (neuere Zahlen liegen nicht vor). ${ }^{20}$ Auseinandersetzungen gab und gibt es auch in anderen Städten, wie z.B. in Stavropol, wo Hunderte von Einheimischen in einer Demonstration die Ausweisung aller »Nichtrussen « aus der Stadt forderten. Die Polizei stand dabei und griff nicht nur nicht ein, sondern ergriff offen Partei für die gewalttätigen Demonstranten. Anlass war die Ermordung von zwei russischen Studenten, angeblich durch Tschetschenen. ${ }^{21}$ Auch ausländische Studenten, insbesondere aus Schwarzafrika und China, sind beliebte Angriffsziele jugendlicher Rowdies.

Als ein Mittel zur Befriedung der Gegensätze wurde u. a. die Schaffung von »ethnischen Abteilungen der Polizei diskutiert «, die in Regionen mit hoher Konzentration von Ausländern zum Kampf gegen organisierte und wirtschaftliche Kriminalität und Extremismus eingesetzt werden sollten. Dieser Gedanke wurde jedoch bald wieder verworfen. Es wurde argumentiert, dass die »ethnische« Polizei mit Sicherheit bald korrumpiert sei, und die Kriminellen eher schützen als verfolgen werde. Um ihren kriminellen Geschäften ungestört nachgehen zu können, versuchen kriminelle Elemente sich bei der Polizei einzukaufen. Das geschieht insbesondere, wenn es darum geht, den eigenen Einfluss auf den großen Märkten zu sichern. Laut Gesetz sollen für den Dienst in der Polizei nur Bürger der Russländischen Föderation mit einer entsprechenden Ausbildung eingestellt werden. Doch gelten sowohl die Polizei, als auch die Untersuchungsbehörden und die Organe der Rechtspflege, obwohl sie fast ausschließlich mit Russen besetzt sind, in der Bevölkerung als besonders korrupt. ${ }^{22}$ Sie sind daher keine Garanten einer öffentlichen Sicherheit.

Wenn man von den anhaltenden kriegsähnlichen Verhältnissen in den kaukasischen Teilrepubliken und den Vorfällen auf Moskaus Manegeplatz im Dezember 2010 absieht, hat es bisher, soweit bekannt, keine größeren Zusammenstöße zwischen der russischen Bevölkerung und den Zugewanderten gegeben. Was jedoch bisher an Gewalttaten (von beiden Seiten) bekannt geworden ist, zeigt, dass die Zuwanderung, auch wenn sie großenteils nur temporärer Art ist, eine Gefährdung der inneren Sicherheit in Russland darstellt. Umso unverständlicher ist es, dass es eine Integrationspolitik in Russland bisher de facto nicht gibt. Auch im Rahmen einer Eurasischen Wirtschaftsunion wird sich dies kaum ändern, da die Mehrzahl der Zuwanderer nicht aus den Staaten der künftigen

19 Z. B. Nezavisimaja Gazeta vom 17. 12. 2010.

20 Ukolov, Roman: Krizisnyj katalisator ksenofobii (Krisisfaktor der Fremdenfeindlichkeit). In: Nezawisimaja gazeta, 28. 1. 2009 (www.ng.ru/moscow/2009-01-28/8_xenophobia.html.).

21 Radio Free Europe, 7. 6. 2007 (www.rferl.net/featuresarticle/2007/06/567245cc-8612-4c9cae.).

22 www.newsru.com/russia/17nov 2011/police_print.html.

ZfP 59. Jg. 3/2012 
Asiatischen Wirtschaftsunion kommt, sondern aus Usbekistan und anderen zentralasiatischen Ländern und Aserbaidschan stammen.

\section{Der Ferne Osten und die chinesische Zuwanderung}

Die Abwanderung von Russen aus dem Fernen Osten des Landes führt zu einer zunehmenden Entleerung der Region. Dies haben die Menschen in den angrenzenden Regionen Chinas, insbesondere aber in den weniger entwickelten Regionen Chinas, sehr bald wahrgenommen und stoßen dort, wo die Russen gehen, nach. Allein in das russische fernöstliche Küstengebiet wandern jährlich ca. 100.000 Chinesen zu, die dort Arbeit suchen und sie, da sie relativ billige Arbeitskräfte sind, auch finden. Die alt eingesessenen Russen dagegen sind wegen des Verlustes ihres Arbeitsplatzes gezwungen, die Gegend zu verlassen. Laut Auskunft des Gouverneurs der fernöstlichen Region wird diese jährlich von mehr als 6000 Menschen verlassen. Der Drang in den russischen Fernen Osten ist nicht zuletzt in der hohen Siedlungsdichte des chinesischen Grenzgebietes am Amur begründet, in dem nicht genügend Arbeitsplätze zur Verfügung stehen. Die Bevölkerungsdichte ist dort zehnmal höher als auf der russischen Seite. ${ }^{23}$

Der Einsatz der kostengünstigen chinesischen Arbeitskraft verbilligt die Produkte der Region und der Investitionsobjekte. So kostet die Verlegung von einem Kilometer Rohrleitung des einer chinesischen Firma übertragenen Abschnitts der Rohrleitung Ostsibirien-Stiller Ozean beim Einsatz russischer Kräfte 18 Mill. Rubel, die Chinesen erledigen die Arbeit für 14 Mill. Rubel. Allerdings setzen die beauftragten chinesischen Firmen allein ihre chinesischen Arbeitskräfte ein, die sich häufig als nicht ausreichend qualifiziert erweisen, so dass sie die ihnen übertragenen Arbeiten nicht den Anforderungen entsprechend ausführen können. Die gesetzten Fristen und technologischen Normen werden oft nicht eingehalten. Der Vertrag wurde gekündigt, dann aber aus Kostengründen wiederbelebt. Die russischen qualifizierten Arbeitskräfte aber wurden verdrängt. Hier ergab sich ein Konflikt zwischen dem Ziel, die Russen in der Region zu halten, und dem Wunsch, den Kostenvorteil, den die Auftragsvergabe an eine chinesische Firma bot, zu nutzen. ${ }^{24}$

Wie viele Chinesen in Russland leben, weiß niemand genau, die Auskünfte der verschiedenen Institutionen gehen daher weit auseinander, was nicht zuletzt politische Gründe hat. Die zentralen Behörden halten die von ihnen gegebenen Zahlen bewusst niedrig, um die ohnehin in Hinblick auf die chinesische Zuwanderung beunruhigte russische Bevölkerung nicht noch mehr zu beunruhigen. Sehr drastisch thematisiert die Zeitung »Izvestija « die bestehenden Ängste: »In zehn Jahren wird der Ferne Osten von

23 Filimonov. Dmitrij: Kitajzy idut (Die Chinesen kommen); in: Izvestija v. 13. 12. 2005 (www.izvetija.ru/special/article3033246.).

24 Saltykova, Natalja: Tschuchie sredi svoich - Kitaiskie migranty wytesnjajut rossijan s rabotschich mest (Fremde unter den Unseren - Die chinesischen Migranten verdrängen die Russen von ihren Arbeitsplätzen): Nesavisimaja Gazeta, 25. 10. 2007 (wwww.ng.ru/2007-10-25/8_aliens.html).Im gegebenen Fall geht es um 1700 Arbeitsplätze. 
Chinesen besiedelt sein und dann wird er insgesamt an China übergehen. $\ll^{25}$ Und noch drastischer: »In einigen Jahrzehnten wird die Ostgrenze Russlands am Ural verlaufen «. ${ }^{26}$ In der »Izvestija« zitierte Schlagzeilen anderer Zeitungen lauten: »Die Chinesen sind unbewaffnet, aber gefährlich «, oder: »Der ,Große Bruder' greift nach uns « oder: »Wird Vladivostok eine Vorstadt von Harbin? « und schließlich: »Die Chinesen im Fernen Osten: Sind sie Gäste oder die Herren? «. ${ }^{27}$ Die Chinesen drängen ganz besonders stark in das Gebiet Chabarowsk am Amur. Der asiatische Teil der ehemaligen Sowjetunion wird also zunehmend entrussifiziert. Die Izvestija zitiert Wissenschaftler, die darauf hinweisen, dass »die friedliche ökonomisch-demographische Expansion Chinas für Russland zu einem geopolitischen Problem wird $\ll^{28}$ Dies erschwert zweifellos die von der russischen Regierung angestrebte Wiederherstellung der früheren weltpolitischen Machtposition Russlands, zumal auch ein wahrer Exodus der russischen Bevölkerung aus den ehem. Sowjetischen Republiken Zentralasiens zu beobachten ist.

Offizielle Zahlen aus dem Jahr 2004 besagen, dass 35.000 Chinesen ständig auf dem Territorium der RF leben. Die Gesamtzahl belaufe sich zum gegebenen Moment auf 200.000. Nach Angaben des Innenministeriums sind es $\$ 400$ bis 700 tausend «. Russische Zeitungen sprechen für das genannte Jahr jedoch von 3-12 Millionen. Die Hälfte der im Gebiet des Fernen Ostens lebenden Chinesen, so die Zeitung »Izvestija«, lebt dort illegal. Sie überschreiten die Grenze mit einem Visum für dreißig Tage, »vergessen« dann aber in ihre Heimat zurückzukehren..$^{29}$ Sie leben in improvisierten Erdhütten und Baracken, sind geschäftstüchtig und fleißig und sind, so lange sie illegal bleiben, kostengünstig. Viele heiraten russische Frauen und legalisieren sich dadurch. Die Frauen nehmen sie gerne, da sie, im Gegensatz zu vielen russischen Männern, zumeist arbeitsam sind und nicht oder nur wenig trinken.

Obwohl einsichtig ist, dass der russische Ferne Osten die Zuwanderung von Arbeitskräften dringend benötigt, ist die Ablehnung der Chinesen durch die russische Bevölkerung fundamental. Der Ferne Osten, einschließlich der Insel Sachalin, und das nördliche Sibirien haben seit 1989 zwischen 20 und 50 Prozent ihrer Bevölkerung verloren. Die Zuwanderung nichtslawischer Völkergruppen führt aber, wie bereits angesprochen, zu sozialen Spannungen. Dies betrifft, wenn von der Migration aus dem Kaukasus abgesehen wird, besonders die Zuwanderung aus China und Vietnam. Die Furcht, von den Chinesen eines Tages dominiert zu werden und den russischen Fernen Osten an die Chinesen zu verlieren, heizt die antichinesische Stimmung an. Tatsächlich ist in einzelnen Kleinstädten und Dörfern der chinesische Bevölkerungsanteil inzwischen derart groß, dass Straßen und Plätze chinesische Namen erhalten haben. In einer Meinungsumfrage aus dem Jahr 2004 spricht sich die Mehrzahl der Befragten dafür aus »den Zuzug nationaler Gruppen« einzuschränken. Das betrifft nicht nur die »Kaukasier « (60 Prozent von 2533 Befragten), sondern auch die Chinesen (51 Prozent) und Vietnamesen (48 Pro-

25 Izvestija, 20. 1. 2006.

26 Izvestija, 20. 9. 2003.

27 Filomonov, Dimitrij: Kitajzy idut (Die Chinesen kommen). Izvestija, 13. 12. 2005.

28 Ebenda.

29 Filomonov, Dimitrij: Kitajzy idut (Die Chinesen kommen), Izvestija, 13. 12. 2005.

ZfP 59. Jg. 3/2012 
zent). ${ }^{30}$ Doch sind es nicht nur nationale oder nationalistische Motive, die der Ablehnung einer Zuwanderung zugrunde liegen. Vielmehr ist es auch »Futterneid «, denn die Gastarbeiter sind zumeist fleißige Arbeiter, die trotz Lohndiskriminierung angeblich oft einen höheren Lebensstandard erreichen als die Russen. Von diesen vertritt laut Umfrage die Mehrheit die Meinung, dass »42 Prozent der Russen in Russland schlechter leben als die Angehörigen der anderen Völkerschaften«. Aus diesem Grund ist es, wie die Zeitung »Izvestija « unter Berufung auf das Forschungszentrum »Levada «schrieb, nicht verwunderlich, dass die öffentliche Meinung nicht mit der regierungsamtlichen übereinstimmt. Diese besagt nämlich, dass als Folge der demographischen Entwicklung die Hinzuziehung ausländischer Arbeitskräfte unumgänglich sei. Nur 13 Prozent der russischen Bevölkerung ist laut der Befragung durch »Levada « der Ansicht, dass die Regierung den legalen Zuzug ausländischer Arbeitskräfte stimulieren sollte. ${ }^{31}$

Im Hinblick auf den Fernen Osten des Landes und die dort zunehmende Immigration chinesischer Zuwanderer versucht die russische Regierung mit einem speziellen, im Jahr 2006 verabschiedeten und vom damaligen Präsidenten Putin verkündeten Umsiedlungsprogramm gegenzusteuern, das die Umsiedlung von dreizehn Millionen Russen in diese Region vorsieht. Das Entwicklungsprogramm für die Fernost-Region und Transbaikalien wurde allerdings zunächst aufgeschoben, so dass sich die Behörden des Gebiets zunächst auf ein vom Zentralkomitee der Kommunistischen Partei im Jahr 1987 (!) verabschiedetes Programm stützen mussten. ${ }^{32}$ Dabei ist die Entwicklung Ostsibiriens und des Fernen Ostens, nicht nur aus demographischen Gründen von besonderer Bedeutung, sie ist vielmehr auch Voraussetzung für eine Beibehaltung des gegenwärtigen Entwicklungstempos, da nur sie eine Aufrechterhaltung des Fördervolumens von Erdöl und Erdgas durch die Erschließung neuer Erdöl- und Erdgasvorkommen ermöglicht. Wie an anderer Stelle erwähnt, gehen die bisher im Westen des Landes ausgebeuteten Ressourcen ihrer Erschöpfung entgegen. ${ }^{33}$

Die Abnahme der Zahl der in diesem Gebiet lebenden Russen findet ihre Begründung nicht nur in einer Abwanderung wegen der herrschenden Lebensumstände, sondern auch in einer relativ hohen Sterblichkeit, die die Geburtsziffern weit übersteigt. Eine »innere Umverteilung und eine Festigung der Kader aus anderen Subjekten der Russländischen Föderation, und eine Erhöhung der Qualifikation der Arbeitskräfte« ist die in einem »Programm für die Entwicklung der Region des Fernen Ostens bis zum Jahr 2025 « vorgeschlagene Gegenstrategie. Da mit Zwangsmaßnahmen im Stil des Stalinismus nicht mehr gearbeitet werden kann und auch die »Neuland-Kampagne Chuschtschovs der fünfziger Jahre kein Vorbild sein kann (es ging um die Urbarmachung der mittelasiati-

30 Ilicev, Georgij: Ocenj mnogo razvelos jushnych nazionalnostej (Es ist sehr viel, was die südlichen Nationalitäten ins Haus gebracht hat), in: Izvestija, 28. 1. 2005.

31 Iljicev, Georgij, a. a. O.

32 Nezavisimaja Gazeta, 4. 6. 2007.

33 Vgl. hierzu Gumpel, Werner: Russland - Quo vadis? Zur wirtschaftlichen und sozialen Zukunft Russlands. In: Politische Studien, 58. Jg., Nr. 415, September/Oktober 2007, insbes. S. $37 \mathrm{ff}$. 
schen Steppen), ${ }^{34}$ sollen jetzt vermehrt materielle Anreize eingesetzt werden. Ein Beispiel hierfür ist die kostenlose Landvergabe an Neusiedler in den wenig erschlossenen Gebieten des Fernen Ostens und der Baikalregion. Ob hier allerdings die Vergabe von 0,3 Hektar pro Familie »für den individuellen Wohnungsbau « ausreichend ist, darf bezweifelt werden. ${ }^{35}$ So lange nicht ausreichende Lebensbedingungen geschaffen sind, wozu insbesondere ein funktionierendes Gesundheits- und ein Bildungswesen und insbesondere Wohnungen gehören, werden sich vermutlich nur wenige Menschen finden, die freiwillig in die Erschließungsgebiete ziehen. Zunächst sind daher Investitionen in Milliardenhöhe erforderlich, die jedoch, nach allen bisherigen Erfahrungen lange auf sich warten lassen werden.

\section{Folgerungen}

Die externe Migration in die Russländische Föderation führt besonders in Regionen mit starker Abwanderung der Bevölkerung und in Entwicklungsgebieten zu einer Entlastung des Arbeitsmarktes. Dies gilt besonders für den Sektor der unqualifizierten Arbeit, wo Russen nicht mehr tätig werden wollen, oder wo eine Ausbeutung durch Niedriglohn oder Lohnbetrug möglich ist. Die zunehmend perzeptierte Überfremdung des Landes mit Menschen aus Zentralasien und China, aber auch aus dem Kaukasus (Südkaukasus und zu Russland gehörige kaukasische Republiken) führt jedoch zu Friktionen und Zwist zwischen Russen und Zuwanderern, die immer häufiger zu gewalttätigen Auseinandersetzungen auch größeren Maßstabs führen. Diese stellen eine Gefährdung der inneren Sicherheit Russlands dar. Da die Immigration zum größten Teil auf Armut beruht, nimmt auch die Leicht- und Schwerkriminalität zu. Die Zuwanderer werden dabei von Gruppierungen der russischen Mafia häufig für deren Zwecke, z. B. für Auftragsmorde und andere Anschläge, missbraucht. Dies alles erhöht die Ausländerfeindlichkeit im Lande und stärkt die nationalistischen Kräfte. Rechtsextreme nutzen dieses Klima, indem sie gezielt und ungezielt Ausländer überfallen, verprügeln und häufig ohne wirklichen Anlass ermorden. Der innere Frieden ist besonders in den dicht besiedelten Städten und Regionen gefährdet.

Relativ friedlich verläuft dagegen, soweit dies aus den Massenmedien zu entnehmen ist, das Zusammenleben der russischen mit der chinesischen Ethnie in den von chinesischer Zuwanderung betroffenen Gebieten. Hier aber handelt es sich nach weit verbreiteter Ansicht um eine »friedliche Landnahme«, die die Integrität des russischen Staates gefährdet. Es fragt sich, wie lange und in welchem Ausmaß die russische Regierung dies hinzunehmen bereit ist, und ob hier nicht ein Konfliktpotential gegenüber der Volksrepublik China im Entstehen ist.

Ein besonderes Problem stellen die in den vergangenen Jahren immer wieder verübten Terroranschläge in russischen Städten (insbesondere in Moskau) und gegen Transport-

34 Vgl. hierzu Nove, Alec : Die Agrarwirtschaft. In :Osteuropa-Handbuch Sowjetunion. Das Wirtschaftssystem, Köln Graz 1965, insbes. S. 357 ff.

35 Vgl. www.realty.newsru.com/article/20.jan2010/30sotok.

ZfP 59. Jg. 3/2012 
einrichtungen (Bahn, Rohrleitungen) dar. Hier wird die Schuld zwar nicht den Immigranten zugeschoben, sondern zumeist Tschetschenen und Inguschen. Terroristische Anschläge gibt es aber auch in den zur RF gehörenden kaukasischen Republiken Tschetschenien, Inguschetien und Daghestan. Auch wenn es sich bei den Beschuldigten nicht um Migranten im hier verwendeten Sinn des Wortes handelt, bewirken auch sie eine Destabilisierung des Landes, und dies nicht nur durch die Tat an sich, sondern auch durch den durch sie gesteigerten Hass auf die »Nichtrussen «. Unter dem Tarnmantel des Gastarbeiters können Terroristen ohne aufzufallen in die zentralen Gebiete des Landes geschleust werden. Ein wirkliches Konzept zur Bekämpfung des Terrorismus fehlt jedoch bisher. Der auf der anderen Seite auch gegen Nichtbeteiligte ausgeübte Terror des Staates, der sich in rücksichtsloser Verfolgung und Vernichtung oft auch absolut Unschuldiger manifestiert, stärkt wiederum das Terrorpotential unter den Gegnern des Landes und führt zu weiterer Radikalisierung. Ein circulus vitiosus.

Wie bereits erwähnt, gibt es für die Integration der Zuwanderer in die Gesellschaft und die Eingliederung der illegalen Migranten in den Produktionsprozess bisher kein wirkliches Konzept. Ein sogenanntes »Toleranzprogramm « aus dem Jahr 2000, das »Ziele für Toleranz und vorbeugende Maßnahmen gegen Extremismus in der russischen Gesellschaft « formuliert hat, blieb in seien Formulierungen vage, dies gilt auch für eine Reihe anderer auf föderaler oder regionaler Ebene verkündete Toleranzprogramme, die aber nur der Bekämpfung der Fremdenfeindlichkeit, nicht jedoch der Integration dienen wollen. Wirklichen Integrationskonzepten würde wegen der nach wie vor überbordenden Korruption auf allen staatlichen und nichtstaatlichen Ebenen aber auch kein Erfolg beschieden sein. Sowohl die legale als auch die illegale Immigration bleiben für Russland eines der gravierendsten innenpolitischen Probleme der Gegenwart wie auch der weiteren Zukunft.

\section{Zusammenfassung}

Russland verzeichnet eine starke Migrationsbewegung. Dabei handelt es sich sowohl um eine interne Migration aus unterentwickelten und klimatisch benachteiligten Regionen in die zentralen Regionen des Landes als auch um die Zuwanderung aus dem »Nahen Ausland « (ehemalige Sowjetrepubliken) und anderen, vorwiegend asiatischen Staaten. Letztere erfolgt in einem erheblichen Maße illegal. Die von den Russen perzeptierte Überfremdung führt zu Friktionen, die u. a. zu offenen Auseinandersetzungen führen. Besonders problembeladen ist die chinesische »Landnahme «im Fernen Osten Russlands. Die Immigration führt zu Destabilisierung und Schwächung des Landes sowohl nach innen als auch nach außen. Auch die legale Immigration wirkt in diese Richtung.

\section{Summary}

Russia is seeing significant migration, both in the sense of internal migration from underdeveloped and climatically disadvantaged regions to the country's central regions and 
immigration from the »near abroad « (the former Soviet republics) and other, primarily Asian states. A considerable proportion of the latter takes the form of illegal migration. Russians feel that their country is being swamped by foreigners, resulting in frictions which in some cases spark open altercations. The Chinese »land grab « in Russia's far east is particularly fraught with problems. Immigration is destabilizing and weakening the country, both internally and externally. Even legal immigration is having a similar effect.

Werner Gumpel, Migration and Immigration: A Political, Economic and Social Problem in Russia

\section{Freie Berufe}

Aktuelle Forschungsergebnisse für Wissenschaft, Praxis und Politik

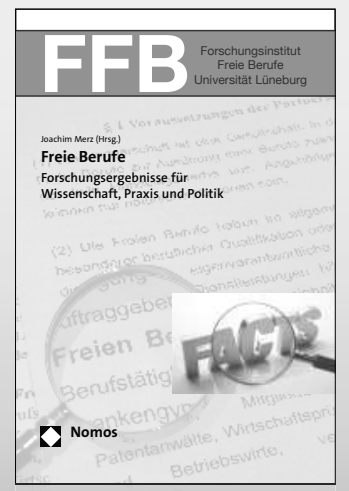

\section{Freie Berufe}

Forschungsergebnisse für Wissenschaft, Praxis und Politik

Herausgegeben von Joachim Merz

2012, 270 S., brosch., 49,- $€$

ISBN 978-3-8329-7846-4

(FFB - Schriften des Forschungsinstituts Freie Berufe der Leuphana Universität Lüneburg, Bd. 20)

20 Jahre Forschungsinstitut Freie Berufe (FFB) der Leuphana Universität Lüneburg: Der Tagungsband Freie Berufe - Forschungsergebnisse für Wissenschaft, Praxis und Politik präsentiert empirisch fundierte, aktuelle Forschungsergebnisse zu Freien Berufen und Einkommen, Zufriedenheit, Berufsverbänden und zu Europa.

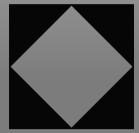

